\title{
Welcome to the 11th Volume of Biomarkers in Medicine
}

Keywords: biomarker $\bullet$ diagnosis $\bullet$ disease $\bullet$ indicator $\bullet$ medicine $\bullet$ predictive $\bullet$ prognosis - research

To all of our readers, we are delighted to welcome you to volume 11 of Biomarkers in Medicine. In this foreword we will present some highlights from the last 12 months in addition to looking forward to the year ahead.

We would also like to take this opportunity to thank all of our valued editorial board members, readers, authors and peer reviewers for their continued support; we very much look forward to seeing the journal progress and develop as we move into 2017.

\section{Content highlights}

Volume 10 saw publication of two wellreceived special focus and themed issues. Back in June 2015, volume 10 issue 6 was entitled Biomarker Discovery: When Wet Meets Dry and guest edited by Hao-Teng Chang (China Medical University, Taiwan). A first for the journal, the issue focused on the discovery of biomarkers or biomarker panels using dry-lab, computational technologies, which provide rapid, systemic calculation, comprehensive combinatory network information and then could accelerate the efficiency with which biomarkers can be applied to clinical practice [1].

Later in the year, a themed issue centered on the latest work in the field of biomarkers for diabetes. Guest edited by editorial board member Jens Peter Goetze (University of Copenhagen, Denmark) and his colleague David P Sonne (also University of Copenhagen), the issue focused on biomarkers being investigated for their potential utility in clinical diabetes assessment and management [2].
At the time of writing our most read article from 2016 was a review entitled 'Value of cerebrospinal fluid $\alpha$-synuclein species as biomarker in Parkinson's diagnosis and prognosis' [2]. The paper, authored collaboratively by authors from University of Perugia (Italy) and University of Gothenburg (Sweden), reviews the clinical evidence for sampling of $\alpha$-synuclein from cerebrospinal fluid in Parkinson's disease. The authors examine both methodological issues and potential diagnostic value, giving recommendations for the future of the approach.

Our most popular research article from the last year was a piece from the aforementioned wet-dry special focus issue, which investigated the correlation between platelet distribution width and chronic obstructive pulmonary disease patients with pulmonary embolism [2].

Another popular article from this volume was an editorial from Urszala Wojda (Nencki Institute of Experimental Biology, Poland), which considered the potential of lymphocytes as biomarkers in Alzheimer's disease [3]. Alzheimer's and other neurodegenerative disorders will continue to be an area of focus for Biomarkers in Medicine in 2017, with the need for earlier diagnosis and ability to monitor disease progress a growing public health concern.

Continuing this theme, our top-scoring paper on the Altmetric platform this year was an editorial article entitled 'Paradigm shift: semantic memory decline as a biomarker of preclinical Alzheimer's disease' [4]. The attention this article received across social media and news outlets further highlights dementia as a priority topic area for the field.

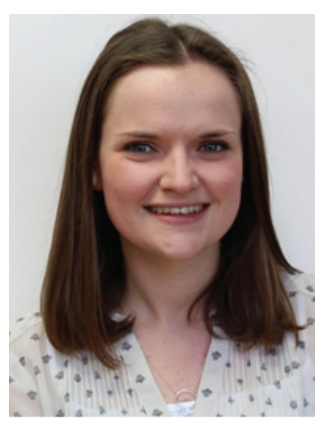

Hannah Wilson

Future Science Group, Unitec House, 2 Albert Place, London N31QB, UK hannah.wilson@futuremedicine.com 


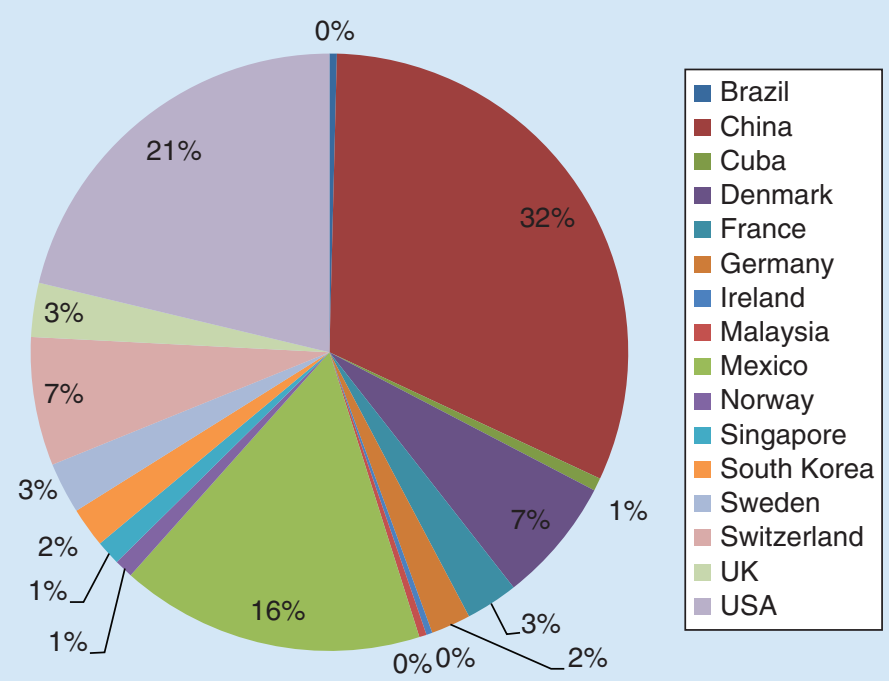

Figure 1. Proportion of readership demographics for Biomarkers in Medicine in 2016.

Other papers to have received high Altmetric scores this year include the following:

- Prognostic biomarker value of binary and grayscale breast tumor histopathology images (Rajkovic et al. [5]).

- Can mean platelet volume and red blood cell distribution width show disease activity in rheumatoid arthritis? (Tecer et al. [6]).

- Identification of Apo B48 and other novel biomarkers in amyotrophic lateral sclerosis patient fibroblasts (Narayan et al. [7]).

- Lysyl oxidase isoforms in gastric cancer (Anazco et al. [8]).

\section{Readership demographics}

As the diversity of the biomarker research community continues to grow, the readership of Biomarkers in Medicine has also changed. In 2016, our content was most read by experts in China (32\%), USA (21\%) and Mexico (16\%) (Figure 1). While our content continues to have impact across Europe and the West, we expect to see continued growth in emerging scientific communities and hope to continue our work toward reaching a truly globalized audience.

\section{References}

1 Chang HT. Biomarker discovery using dry-lab technologies and high-throughput screening. Biomark. Med. 10(06), 559-561 (2016).

\section{Social media}

Biomarkers in Medicine is active across social media and you can follow our latest updates on LinkedIn [9] and at @ fsgbmm on Twitter [10]. We regularly post journal highlights including newly published content and the latest news in the field. We have thoroughly enjoyed engaging with our readers and authors on these platforms and look forward to doing so more in the future.

\section{Conclusion}

We appreciate all feedback on the direction of our content, especially suggestions of any priority topics for the journal to consider. We welcome unsolicited research, review and opinion article proposals, and would be delighted to hear from you if you are interested in submitting to the journal.

We greatly look forward to collaborating with you all over the next year and hope to see Biomarkers in Medicine continue to grow and serve the community in 2017.

\section{Financial \& competing interests disclosure}

$\mathrm{H}$ Wilson is an employee of Future Science Ltd, publisher of Biomarkers in Medicine. The author has no other relevant affiliations or financial involvement with any organization or entity with a financial interest in or financial conflict with the subject matter or materials discussed in the manuscript apart from those disclosed.

No writing assistance was utilized in the production of this manuscript.

2 Goetze JP, Sonne DP. Diabetes and its lack of causal biomarkers. Biomark. Med. 10(11), 1121-1123 (2016).

3 Wojda U. Alzheimer's disease lymphocytes: potential for biomarkers? Biomark. Med. 10(1), 1-4 (2016). 
4 Venneri A, Mitolo M, De Marco M. Paradigm shift: semantic memory decline as a biomarker of preclinical Alzheimer's disease. Biomark. Med. 10(1), 5-8 (2016).

5 Rajković N, Vujasinović T, Kanjer K, Milošević NT, Nikolić-Vukosavljević D, Radulovic M. Prognostic biomarker value of binary and grayscale breast tumor histopathology images. Biomark. Med. 10(10), 1049-1059 (2016).

6 Tecer D, Sezgin M, Kanik A. Can mean platelet volume and red blood cell distribution width show disease activity in rheumatoid arthritis? Biomark. Med. 10(9), 967-974 (2016).
7 Narayan M, Seeley KW, Jinwal UK. Identification of Apo B48 and other novel biomarkers in amyotrophic lateral sclerosis patient fibroblasts. Biomark. Med. 10(5), 453-462 (2016).

8 Añazco C, Delgado-López F, Araya P. Lysyl oxidase isoforms in gastric cancer. Biomark. Med. 10(9), 987-998 (2016).

9 Linkdin group: Biomarkers in Medicine. www.linkedin.com/groups/8444085

10 Twitter: Biomarkers in Medicine. https://twitter.com/fsgbmm 